\title{
A unique case of ectopic Cushing's syndrome from a thymic neuroendocrine carcinoma
}

\author{
Lima Lawrence ${ }^{1}$, Peng Zhang'2, Humberto Choi ${ }^{2}$, Usman Ahmad ${ }^{3}$, Valeria Arrossi ${ }^{4}$, \\ Andrei Purysko ${ }^{5}$ and Vinni Makin ${ }^{1}$
}

Departments of ${ }^{1}$ Endocrinology, Diabetes \& Metabolism, 2 Pulmonary Medicine \& Critical Care, ${ }^{3}$ Thoracic and Cardiovascular Surgery, ${ }^{4}$ Anatomic Pathology, and 5Diagnostic Radiology, Cleveland Clinic, Cleveland, Ohio, USA
Correspondence should be addressed to L Lawrence Email

lawren14@ccf.org

\begin{abstract}
Summary
Ectopic adrenocorticotropic hormone (ACTH) production leading to ectopic ACTH syndrome accounts for a small proportion of all Cushing's syndrome (CS) cases. Thymic neuroendocrine tumors are rare neoplasms that may secrete ACTH leading to rapid development of hypercortisolism causing electrolyte and metabolic abnormalities, uncontrolled hypertension and an increased risk for opportunistic infections. We present a unique case of a patient who presented with a mediastinal mass, revealed to be an ACTH-secreting thymic neuroendocrine tumor (NET) causing ectopic CS. As the diagnosis of CS from ectopic ACTH syndrome (EAS) remains challenging, we emphasize the necessity for high clinical suspicion in the appropriate setting, concordance between biochemical, imaging and pathology findings, along with continued vigilant monitoring for recurrence after definitive treatment.
\end{abstract}

\section{Learning points:}

- Functional thymic neuroendocrine tumors are exceedingly rare.

- Ectopic Cushing's syndrome secondary to thymic neuroendocrine tumors secreting ACTH present with features of hypercortisolism including electrolyte and metabolic abnormalities, uncontrolled hypertension and hyperglycemia, and opportunistic infections.

- The ability to undergo surgery and completeness of resection are the strongest prognostic factors for improved overall survival; however, the recurrence rate remains high.

- A high degree of initial clinical suspicion followed by vigilant monitoring is required for patients with this challenging disease.

\section{Background}

Cushing's syndrome (CS) results in hypercortisolism as a result of excess glucocorticoid production from endogenous sources (adrenals, pituitary, ectopic) or exogenous administration. The incidence of endogenous CS varies from 0.2 to 5 cases per million per year, with a median age of onset around 41.4 years and a female preponderance in a 3:1 ratio (1). CS may be ACTH-dependent or ACTHindependent from adrenal origin. Cushing disease (CD) due to an ACTH-secreting pituitary adenoma are more commonly seen compared to ectopic ACTH syndrome
(EAS), while corticotropin-releasing hormone producing tumors causing CS are exceedingly rare $(<1 \%)$. Liddle and colleagues first described 'ectopic 'adrenocorticotropin' produced by nonpituitary neoplasms as a cause of Cushing's syndrome' in 1962 (2). EAS is most commonly seen in association with small cell lung carcinoma or carcinoid tumors originating in the lungs or gastrointestinal tract. While the clinical presentation of CS is highly variable making diagnosis challenging, it is crucial that patients with EAS are identified and treated in a timely manner. 
They have features of severe hypercortisolism and have a florid presentation with significant derangements in electrolytes, uncontrolled hypertension and hyperglycemia, with an increased risk for life-threatening opportunistic infections. Herein, we present a unique case of ectopic CS caused by an ACTH-secreting thymic NET, initially misdiagnosed as a benign lipoma.

\section{Case presentation}

A 54-year-old gentleman presented to the emergency department with new-onset oral thrush and right-sided facial swelling after dental work. He had extensive recent evaluation for various complaints including weight gain, near-syncopal episodes, pleuritic chest pain and exertional dyspnea. Eight months ago, patient presented to his dermatologist with increasing photosensitivity and darkening of the skin. A high ACTH level was noted, which led to additional evaluation with $24 \mathrm{~h}$ urinary free cortisol (Table 1). The patient was referred to endocrinology for further testing but failed to do so. One month after the abnormal adrenal testing results, a chest X-ray following a motor vehicle accident revealed a mediastinal mass measuring approximately $13.5 \times 11.0 \mathrm{~cm}$. Computed tomography (CT) chest confirmed a large anterior mediastinal mass measuring $10.7 \times 6.3 \mathrm{~cm}$ in transverse and anteroposterior dimensions and $11.0 \mathrm{~cm}$ in craniocaudad dimensions, extending above the level of the clavicle and displacing the trachea to the right. No hilar, paratracheal, axillary or subpectoral lymphadenopathy was noted. A biopsy performed at an outside hospital via video-assisted thoracoscopic surgery revealed well-differentiated adipose tissue. However, a question was raised by the pathologist about possible sampling error as the biopsied material could represent normal adipose tissue adjacent to or associated with an unsampled neoplasm.

\section{Investigation}

During the current inpatient admission, patient reported proximal muscle weakness, myalgias and unintentional central weight gain of 25 pounds. Physical exam was remarkable for an obese man with a cushingoid appearance, facial fullness and plethora. He had dorsocervical and supraclavicular fat pads with hyperpigmentation of the sun-exposed regions. No abdominal striae, lower extremity edema or proximal muscle weakness was noted on strength testing. Labs revealed severe hypokalemia refractory to replacement, metabolic alkalosis, rhabdomyolysis and leukocytosis without fever. Given the clinical presentation, labs and history of elevated ACTH with a mediastinal mass, there arose a concern for ectopic ACTH production resulting in CS. Repeat random cortisol and ACTH were elevated and remained non-suppressed after an $8 \mathrm{mg}$ dexamethasone suppression test (Table 1). Prior CT chest images were reviewed to localize the source of ectopic cushings, which revealed that the mediastinal mass lacked fat attenuation, inconsistent with the presumed prior diagnosis of lipoma. Repeat imaging redemonstrated an $11.7 \times 7.9 \mathrm{~cm}$ bilobed anterior mediastinal mass extending above the thoracic inlet with extrinsic compressive effect on the esophagus and trachea. Bilateral hilar and perihilar lymphadenopathy was noted along with new-onset bilateral adrenal hyperplasia (Fig. 1). Wholebody positron emission tomography (PET)-CT revealed a mild-to-moderately fludeoxyglucose (FDG) avid mass extending across the anterior mediastinum, with symmetric thickening and hypermetabolism of the adrenal glands, likely due to adrenal hyperplasia (Fig. 2). Magnetic resonance imaging (MRI) of the pituitary noted normal size and configuration of the pituitary gland without evidence of an adenoma or suprasellar mass (Fig. 3).

Table 1 Trend of pertinent laboratory findings.

\begin{tabular}{|c|c|c|c|c|}
\hline & Reference range & 8 months ago & Hospital admission & $\begin{array}{c}\text { Post } 8 \text { mg dexamethasone } \\
\text { suppression test }\end{array}$ \\
\hline Potassium (mmol/L) & $3.5-5.1$ & & 1.7 & \\
\hline Bicarbonate (mmol/L) & $22-30$ & & 46 & \\
\hline Creatinine kinase (U/L) & $55-170$ & & 4828 & \\
\hline White blood cells $(k / \mu L)$ & $4.5-11.0$ & & 26.58 & \\
\hline Random cortisol ( $\mu \mathrm{g} / \mathrm{dL})$ & $1.7-22.7$ & & 146.9 & 133.9 \\
\hline $\mathrm{ACTH}(\mathrm{pg} / \mathrm{mL})$ & $<47$ & 231 & 1037 & 1135 \\
\hline 24 h urinary free cortisol ( $\mu g /$ day) & $\leq 60$ & 68.1 & & \\
\hline Dexamethasone (ng/dL) & $>200$ & & & 427.0 \\
\hline
\end{tabular}



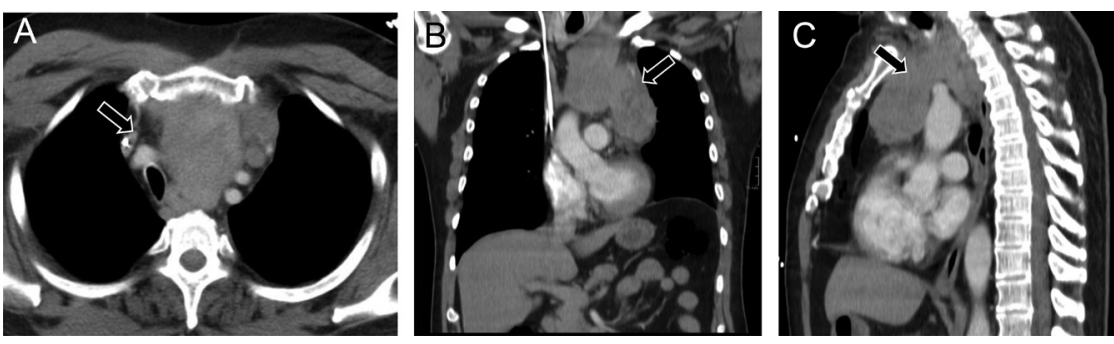

\section{Figure 1}

Chest CT with IV contrast. Axial (A), coronal (B) and sagittal (C) images of the chest demonstrate a bilobed mass (arrow) centered in the anterior mediastinum insinuating into the left paratracheal region displacing the aortic arch branch vessels, and extending in the thoracic inlet.

\section{Treatment}

For treatment of EAS, patient was started on a regimen of ketoconazole $300 \mathrm{mg}$ twice daily and mifepristone $300 \mathrm{mg}$ daily for 1 week prior to surgical removal of the mediastinal mass. A combination regimen of ketoconazole and mifepristone was used in a multipronged approach against hypercortisolism, as ketoconazole inhibits multiple enzymes in cortisol synthesis, while mifepristone exerts its actions against the glucocorticoid receptor. Hypokalemia was aggressively repleted, along with controlling new-onset hypertension and hyperglycemia. Patient underwent surgical removal of the large mediastinal mass along with pericardial resection and wedge resection of the left upper lobe of the lung. In the immediate postoperative period, patient experienced no arrhythmias, but developed hypotension refractory to fluid resuscitation, requiring initiation of vasopressors and intravenous dexamethasone. It is likely that glucocorticoid receptor blockade by mifepristone and inhibition of cortisol synthesis by ketoconazole, along with operative removal of the ACTH source contributed to the resulting adrenal insufficiency. A significant decline in ACTH and cortisol levels was noted postoperatively and the patient was transitioned to oral hydrocortisone.

\section{Outcome and follow-up}

Pathology revealed a large thymic neuroendocrine carcinoma measuring $12.5 \times 8.0 \times 4.5 \mathrm{~cm}$ with less than two mitoses per ten high power fields without necrosis and a Ki proliferative index of 5\%. According to the World Health Organization (WHO) classification of NETs, based on mitotic count and absence of necrosis, the tumor was classified as a typical low-grade carcinoid $(3,4)$. Staging was classified as T3N1M0 due to direct invasion of the tumor into the lung and metastases to regional lymph nodes in the anterior mediastinum. Immunohistochemical staining for ACTH on a single block of tumor was negative; however, it is likely that ACTH is variably expressed in different regions of this large tumor (Figs 4 and 5). As the mass was completely excised with only microscopicpositive margins (R1 resection), postoperative radiation was not recommended as adjuvant treatment. Radiation has not been shown to affect overall survival for resected typical carcinoid tumors, regardless of nodal involvement or tumor size. This collaborative recommendation was made during a multidisciplinary meeting, and active surveillance was initiated.

Patient has had regular follow-up for the past 8 months after hospital discharge, with remarkable improvement in electrolyte abnormalities, weight loss greater than
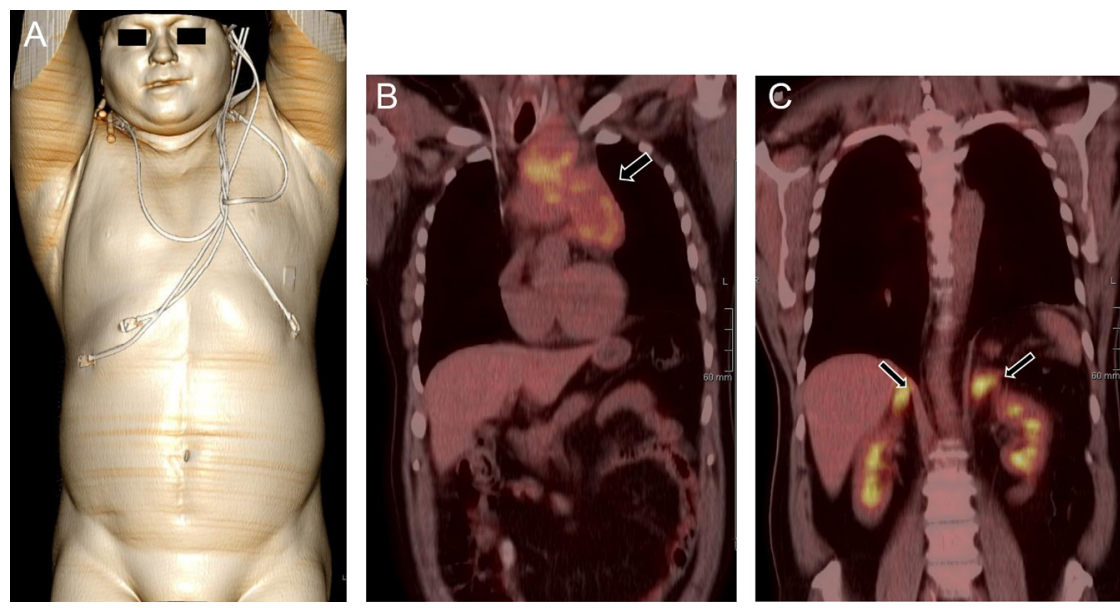

Figure 2

Whole-body 18F-FDG-PET/CT. 3D volumerendered $\mathrm{CT}$ image $(\mathrm{A})$ demonstrate central obesity and cushingoid facies. Fused coronal PET/ $\mathrm{CT}$ image demonstrates moderate and heterogeneous FDG uptake by the mediastinal mass (arrow, B) and intense, symmetric uptake by the adrenal glands (arrows, C). 

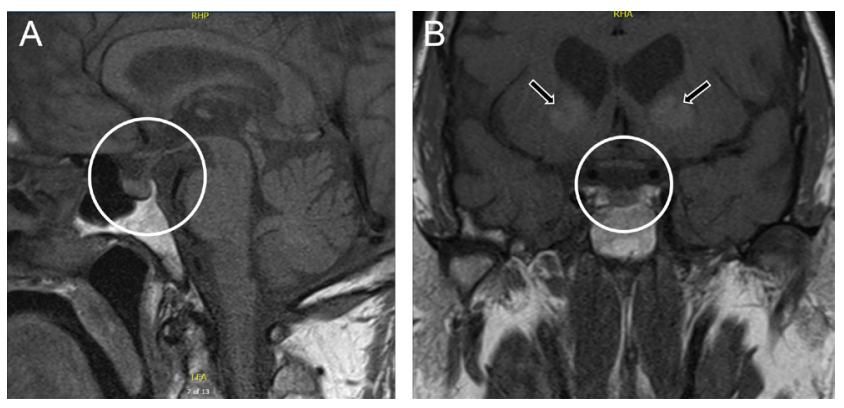

Figure 3

MRI pituitary with and without contrast. The pituitary gland (circled, A) is within normal limits of size and configuration without pituitary adenoma or suprasellar mass. Symmetric calcifications (arrows, B) within the caudate heads, and lentiform nucleus suggests dystrophic calcification.

65 pounds and normalization of blood glucose, blood pressure, ACTH and cortisol levels. He has not required reinitiation of medications for hypertension or glycemic control. Patient has been tapered off hydrocortisone therapy with recovery of adrenal function confirmed on an ACTH stimulation test.

\section{Discussion}

We present a unique case of ectopic CS caused by an ACTH-secreting thymic NET initially misdiagnosed as a lipoma. Thymic NETs are exceedingly rare accounting for $2 \%$ of all mediastinal tumors and $5 \%$ of all thymic tumors. Thymic NETs are three times more commonly seen in men, with a peak incidence around 54 years of age, and are biochemically active in $30-50 \%$ of all cases (5). EAS from thymic NETs are seen in $40-50 \%$ of hormonally active thymic NETs. A meta-analysis of EAS cases reported in literature have suggested a slight male preponderance and earlier presentation in the second and third decades of life (6). EAS from thymic NETs have a worse outcome than biochemically inactive thymic tumors, likely due to its inherent aggressiveness coupled with the devastating metabolic derangements resulting
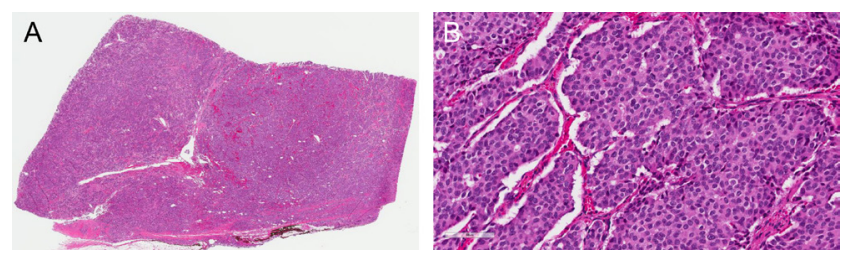

\section{Figure 4}

(A) Low power showing a cellular highly vascularized neoplasm (H\&E, $0.4 \times$ ). (B) Solid nests of polygonal cells with round to slightly oval nuclei with finely granular chromatin, inconspicuous nucleoli and ample eosinophilic cytoplasm. No mitotic figures or necrosis are present (H\&E, $25 \times)$.
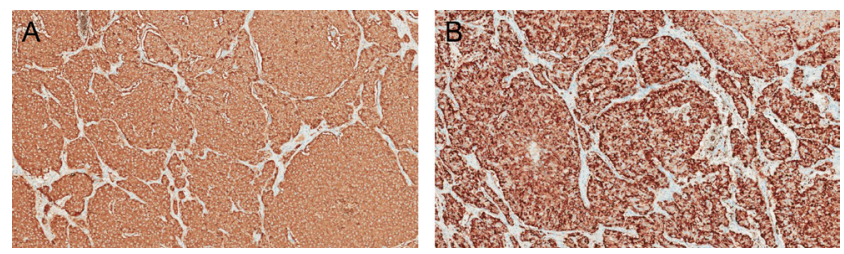

\section{Figure 5}

Immunohistochemical markers confirm neuroendocrine immunophenotype of the tumor cells (A) synaptophysin, (B) chromogranin (10x).

from CS. Patients present with features of severe, rapidonset hypercortisolism including profound hypokalemia due to the action of cortisol on mineralocorticoid receptors, metabolic alkalosis, resistant hypertension, hyperglycemia, hyperpigmentation, edema and proximal muscle weakness, while features of longterm hypercortisolism such as significant weight gain, obesity and violaceous striae are infrequently seen (7). Hypercortisolemia also places these patients at increased risk for bacterial or opportunistic infections, as noted in our patient who presented with oral thrush in the absence of human immunodeficiency virus infection (8).

Investigation of EAS begins with clinical suspicion confirmed with biochemical evidence of hypercortisolism and elevated ACTH, followed by localization of the source: pituitary versus ectopic. Various case series have established that an ACTH level $>200 \mathrm{pg} / \mathrm{mL}$ suggests EAS over CD (9). Imaging studies including CT, ${ }^{18} \mathrm{FDG}$ PET or ${ }^{68} \mathrm{Ga}$-DOTATATE PET are useful in visualizing the extent of tumor and evaluating metastatic disease. Complete R0 surgical resection is the only curative method, while the ability to undergo surgery and completeness of the resection are the strongest prognostic factors for improved overall survival (5). Unfortunately, even with radical surgery and lymph node dissection, the recurrence rate remains high. Incompletely resected tumors may be treated with postoperative chemotherapy and radiation; however, no statistically significant improvement in outcomes are seen with adjuvant therapy. Therefore, patients with EAS from thymic NETs have a challenging condition that need aggressive surgical resection followed by close monitoring to evaluate for recurrence of disease.

Our patient was initially found to have biochemical evidence of hypercortisolism and failed to follow-up with endocrinology as recommended. Subsequently, a large mediastinal mass was discovered and falsely identified as a lipoma due to sampling error on biopsy. Finally, eight months after detection of elevated cortisol, and 
six months after discovery of the mediastinal mass, the patient underwent definitive surgical resection of the thymic NET causing ectopic CS. There are several unique features of this case that warrant emphasizing. The mediastinal mass was falsely identified as a lipoma due to sampling error on biopsy in spite of the biochemical evidence of hypercortisolism, and imaging characteristics on CT where the mediastinal mass lacked fat attenuation, inconsistent with the presumed diagnosis of lipoma. This highlights the need for a high clinical suspicion for CS in the appropriate setting and to evaluate the biochemical, imaging and pathology results cohesively to ensure the findings lead to a unified diagnosis. On initial presentation, the patient was found to have severe electrolyte abnormalities including refractory hypokalemia and metabolic alkalosis. Preoperative management of the patient included addressing the hypercortisolemia with ketoconazole and mifepristone. This combination therapy is typically contraindicated as both drugs can lead to QT prolongation and strong inhibition of CYP3A by ketoconazole, which increases the levels of mifepristone in the blood (10). However, concurrent therapy in this patient was medically necessary to rapidly ameliorate severe hypercortisolism, and patient was monitored very closely for medication tolerability in an inpatient setting. Although medical treatment of CS is possible by inhibition of ACTH secretion (octreotide, pasireotide, cabergoline), inhibition of adrenal steroidogenesis (ketoconazole, metyrapone, mitotane, etomidate) or by glucocorticoid receptor blockade (mifepristone), the definitive treatment of ectopic CS is by surgical removal of the tumor. Emergency treatment of severe ectopic CS may require combination therapy or parenteral etomidate under vigilant supervision in an intensive care unit setting. Bilateral adrenalectomy may be considered in select patients with uncontrolled, life-threatening hypercortisolemia refractory to medical management.

We present a case of EAS due to a large thymic NET initially misdiagnosed as a lipoma. Our patient underwent successful surgical resection of the tumor with the development of postoperative adrenal insufficiency and subsequent recovery of adrenal function. As the diagnosis of CS from EAS remains challenging, we emphasize the need for high clinical suspicion in the appropriate setting, confirmation of the diagnosis on biochemical, imaging and pathology findings, along with continued vigilant monitoring for recurrence.

\section{Declaration of interest}

The authors declare that there is no conflict of interest that could be perceived as prejudicing the impartiality of the research reported.

\section{Funding}

This research did not receive any specific grant from any funding agency in the public, commercial or not-for-profit sector.

\section{Patient consent}

The patient has given written informed consent.

\section{Author contribution statement}

All authors contributed equally to the conception, writing and editing of the manuscript. L Lawrence: Endocrinology fellow took care of the patient in the hospital and currently in the outpatient office. $P$ Zhang: Pulmonary-Critical Care fellow took care of the patient in the hospital. $\mathrm{H}$ Choi: Pulmonary-Critical Care attending took care of the patient in the hospital. U Ahmad: Cardiothoracic surgeon performed the surgical resection and currently following the patient in the outpatient office. $V$ Arrossi: Pathologist provided the pathology report and images. A Purysko: Radiologist provided the radiographic findings and images. $\mathrm{V}$ Makin: Endocrinology attending took care of the patient in the hospital.

\section{References}

1 Lacroix A, Feelders RA, Stratakis CA \& Nieman LK. Cushing's syndrome. Lancet 2015386 913-927. (https://doi.org/10.1016/ S0140-6736(14)61375-1)

2 Liddle GW, Island DP, Ney RL, Nicholson WE \& Shimizu N. Nonpituitary neoplasms and Cushing's syndrome. Ectopic 'adrenocorticotropin' produced by nonpituitary neoplasms as a cause of Cushing's syndrome. Archives of Internal Medicine 1963111 471-475. (https://doi.org/10.1001/archi nte.1963.03620280071011)

3 Travis W, Brambilla E, Burke A, Marx A \& Nicholson AG. WHO Classification of Tumors of the Lung, Pleura, Thymus and Heart. Lyon: IARC Press, 2015.

4 Gaur P, Leary C \& Yao JC. Thymic neuroendocrine tumors: a SEER database analysis of 160 patients. Annals of Surgery 2010251 1117-1121. (https://doi.org/10.1097/SLA.0b013e3181dd4ec4)

5 Filosso PL, Yao X, Ahmad U, Zhan Y, Huang J, Ruffini E, Travis W, Lucchi M, Rimner A, Antonicelli A, et al. Outcome of primary neuroendocrine tumors of the thymus: a joint analysis the International Thymic Malignancy Interest Group and the European Society of Thoracic Surgeons databases. Journal of Thoracic and Cardiovascular Surgery 2015149 103.e2-109.e2. (https://doi. org/10.1016/j.jtcvs.2014.08.061)

6 Neary NM, Lopez-Chavez A, Abel BS, Boyce AM, Schaub N, Kwong K, Stratakis CA, Moran CA, Giaccone G \& Nieman LK. Neuroendocrine ACTH-producing tumor of the thymus experience with 12 patients over 25 years. Journal of Clinical Endocrinology and Metabolism 201297 2223-2230. (https://doi. org/10.1210/jc.2011-3355) 
7 Isidori AM, Kaltsas GA, Pozza C, Frajese V, Newell-Price J, Reznek RH, Jenkins PJ, Monson JP, Grossman AB \& Besser GM. The ectopic adrenocorticotropin syndrome: clinical features, diagnosis, management, and long-term follow-up. Journal of Clinical Endocrinology and Metabolism 200691 371-377. (https://doi. org/10.1210/jc.2005-1542)

8 Sarlis NJ, Chanock SJ \& Nieman LK. Cortisolemic indices predict severe infections in Cushing syndrome due to ectopic production of adrenocorticotropin. Journal of Clinical Endocrinology and Metabolism 200085 42-47. (https://doi.org/10.1210/ jcem.85.1.6294)
9 Yogi-Morren D, Habra MA, Faiman C, Bena J, Hatipoglu B, Kennedy L, Weil RJ \& Hamrahian AH. Pituitary MRI findings in patients with pituitary and ectopic ACTH-dependent Cushing syndrome: does a 6-mm pituitary tumor size cut-off value exclude ectopic ACTH syndrome? Endocrine Practice 201521 1098-1103. (https://doi.org/10.4158/EP15662.OR)

10 Fleseriu M, Molitch ME, Gross C, Schteingart DE, Vaughan TB \& Biller BM. A new therapeutic approach in the medical treatment of Cushing's syndrome: glucocorticoid receptor blockade with mifepristone. Endocrine Practice 201319 313-326. (https://doi. org/10.4158/EP12149.RA)

Received in final form 28 January 2019

Accepted 5 February 2019 